\title{
Um Mapeamento Sistemático sobre Sistemas que Envolvem IoT e Deep Learning Conjuntamente
}

\author{
Janyelson V. L. de Oliveira, João L. F. Amorim, Matheus M. R. Praxedes \\ Centro de Informática - Universidade Federal da Paraíba \\ \{janyelsonvictor, jony.lucas.br13, matheuspraxedes2014\}@ gmail.com
}

\begin{abstract}
Resumo: Na última década, a Internet das Coisas (IoT) e Deep Learning receberam significativa atenção da indústria, bem como das universidades e dos centros de pesquisa. As principais razões por trás desse interesse está no fato que IoT permite conectar diversos dispositivos ao nosso redor por meio da Internet - criando ambientes inteligentes - e Deep Learning mostrou-se ter bons resultados em uma série de aplicações, em comparação com as abordagens tradicionais, principalmente, na classificação de imagens. Neste trabalho realizamos um mapeamento sistemático de literatura com o objetivo de identificar como IoT e Deep Learning podem funcionar conjuntamente em diversos sistemas e aplicações. Os resultados apontaram que a união dessas duas abordagens é útil para a solução de vários problemas práticos.
\end{abstract}

Palavras-chave: Deep Learning; IoT; Mapeamento sistemático.

\section{Introdução}

Sistemas IoT (em inglês, Internet of Things) e Deep Learning (em português, Aprendizado Profundo) são termos comuns quando pensamos em tecnologias modernas que utilizam comunicação entre componentes eletrônicos e aprendizagem de máquina, respectivamente. Nos últimos anos, vêm surgindo bastante interesse tanto no desenvolvimento e uso de sistemas baseados em IoT quanto em aplicar técnicas de redes neurais artificiais (ANNs), especificamente Deep Learning, para aprendizagem e predição com o intuito de resolver algum tipo de problema [25,26].

Não há como medir a extensão dos sistemas IoT, pois o termo IoT engloba tecnologias compostas por uma estrutura que permite a identificação e comunicação por meio de radiofrequência (RFID), comunicação por meio da Internet, por protocolos e tecnologias de rede e traz desafios adicionais como armazenamento e compartilhamento de dados [25].

A ideia básica por trás da IoT é conectar todos as coisas ao nosso redor utilizando a Internet, fazendo com que se tenha um ambiente inteligente, onde os diversos dispositivos podem se comunicar uns com os outros de forma semelhante à comunicação entre humanos [8].

Deep Learning é imensamente utilizado em tecnologias que envolvem predição a partir de dados coletados, permitindo o desenvolvimento de tecnologias como filtros na Internet (e.g. filtro de spams), recomendações de produtos, entre outros [26]. Os estudos e o desenvolvimento da aprendizagem profunda possibilitou grandes progressos também nas áreas de reconhecimento de imagens e processamento de voz. As redes neurais de aprendizado profundo são superiores a outros métodos tradicionais de reconhecimento de imagem e tem feito grandes progressos no processamento de dados massivos e na computação distribuída [5].

Neste artigo é realizado um mapeamento sistemático com intuito de encontrar artigos relacionados a sistemas IoT e Deep Learning. O objetivo desse mapeamento é entender como funcionam (métodos, tecnologias, arquitetura, integração, comunicação e princípios) os sistemas que utilizam Deep Learning e IoT conjuntamente, além de buscar as principais aplicações (contextos de uso) desses sistemas.

\section{Metodologia}

O procedimento metodológico escolhido foi o mapeamento sistemático, pois utiliza uma metodologia bem definida que permite identificar e analisar tópicos de pesquisa em diferentes áreas do conhecimento, inclusive em áreas da computação.

O mapeamento sistemático foi feito sobre artigos que envolvem estudos e/ou desenvolvimento de sistemas baseados em IoT e Deep Learning conjuntamente. Diante disso, foram criadas questões que atendessem aos principais objetivos da pesquisa, são eles: descobrir como funcionam os sistemas que utilizam Deep Learning e IoT conjuntamente (principais arquiteturas, tecnologias, princípios e requisitos dos sistemas); e quais as principais aplicações e contextos de uso desses sistemas, como mencionado na seção anterior. O mapeamento sistemático foi executado, em uma visão mais geral, com os seguintes passos:

1. Conhecido o tópico/tema de pesquisa, elaborar questões de pesquisa que deverão ser respondidas ao longo de todo o mapeamento;

2. Elaborar uma string de busca (ou chave de busca) para o tema proposto;

3. Escolher os motores de buscas pelo qual a string será executada e os artigos serão pesquisados;

4. Definir quais serão os critérios de inclusão e exclusão para a seleção dos artigos; e

5. Selecionar nos motores de busca escolhidos, ao longo de várias etapas consecutivas, quais artigos respondem às questões de pesquisa para o tema proposto e que também atendam aos critérios de inclusão e exclusão.

\subsection{Questões de Pesquisa}

A presente subseção mostra quais foram as questões de pesquisa utilizadas ao longo do mapeamento, são elas:

Questão 1: Como a IoT pode contribuir nos sistemas e aplicações que utilizam Deep Learning e vice-versa?

Questão 2: Quais os principais requisitos e princípios aplicados em sistemas que utilizam Deep Learning e IoT conjuntamente?

Questão 3: Qual(is) a(s) principal(is) arquitetura(s) empregada(s) em sistemas que utilizam Deep Learning e IoT conjuntamente? 
Questão 4: Qual o contexto de uso desses sistemas? O objetivo é saber quais são os principais contextos de uso ou quais estudos sobre o tema já estão sendo realizados. Questão 5: Quais exemplos de aplicações/sistemas existentes?

\subsection{Condução da Pesquisa}

Após a definição das questões de pesquisa, a busca e seleção dos artigos foram feitas utilizando dois dos principais motores de busca (ou base de dados) que possuem artigos na área de computação ou informática e que apresentaram melhores resultados de artigos relacionados com Deep Learning e sistemas IoT, são eles: Scopus e Springer.

A string de busca foi elaborada com base nas questões de pesquisa e que apresentasse, ao mesmo tempo, um melhor resultado nos motores de buscas citados. Logo, a string a escolhida foi: ("Internet of Things" OR IoT) AND ("Deep Learning”) - com as devidas modificações de sintaxe para que pudesse ser aceita nas duas bases.

\subsection{Triagem dos Artigos}

Para a seleção dos artigos relevantes para as questões de pesquisa, foram elaborados critérios de exclusão e de inclusão. Os critérios de inclusão escolhidos foram: publicações (entre 2014 e 2018); escritas em português e/ou inglês; com até 20 páginas; e que apresentassem contextos de uso. Entre os critérios de exclusão temos: publicações com até 5 páginas; não foram aceitas publicações duplicadas nem pagas ou não disponíveis nem revisões literárias.

Após a elaboração dos critérios, foram realizados os seguintes passos para a seleção dos artigos: o $1^{\circ}$ passo foi selecionar todos os artigos resultantes da aplicação da string de busca nos motores de pesquisa de cada base de busca; o $2^{\circ}$ passo foi realizar a leitura dos resumos dos artigos selecionados no passo anterior e realizar a primeira triagem de acordo com os critérios de inclusão e exclusão definidos; e, por último, o $3^{\circ}$ passo consistiu na seleção dos artigos após a leitura da sua introdução e que também estivessem de acordo com os critérios de inclusão e exclusão citados.

\section{Resultados e Discussão}

Os resultados foram divididos em resultados gerais da pesquisa (englobando todos os artigos selecionados), seguido pelas respostas aos questionamentos propostos (também de acordo com cada artigo selecionado).

\subsection{Resultados Gerais}

No final, o mapeamento sistemático resultou em um total de 24 artigos. A maior quantidade de publicações ocorreu no ano de 2017 (com 15 artigos), seguido pelo ano de 2016 (com 5 artigos). Já nos anos de 2014 e 2018 foram publicados 1 e 3 artigos, respectivamente. No ano de 2015, não houveram publicações sobre o tema pesquisado nos motores de busca escolhidos.

\subsection{Questão 1: Como a IoT pode contribuir nos} sistemas e aplicações que utilizam Deep Learning $e$ vice-versa?

Por questões de simplificação e de melhor visualização, optamos, primeiramente, apresentar as contribuições de técnicas de Deep Learning para os sistemas IoT. Sendo assim, as contribuições encontradas nos artigos decorrentes do mapeamento sistemático foram sumarizadas no Quadro 1.

Quadro 1: Contribuição de técnicas de deep learning para sistemas IoT.

\begin{tabular}{|l|l|l|}
\hline Contribuição (Deep Learning) & $\#$ & Referências \\
\hline $\begin{array}{l}\text { Processamento e análise dos dados } \\
\text { coletados }\end{array}$ & 8 & {$[3 ; 4 ; 5 ; 16 ; 20 ; 21 ; 22 ; 24]$} \\
\hline Extração de características & 5 & {$[2 ; 14 ; 20 ; 21 ; 23]$} \\
\hline $\begin{array}{l}\text { Reconhecimento/classificação de } \\
\text { padrões visuais por imagens }\end{array}$ & 4 & {$[10 ; 11 ; 18 ; 19]$} \\
\hline Auxílio em tomadas de decisão & 4 & {$[1 ; 2 ; 4 ; 9]$} \\
\hline Melhorar o tráfego da rede & 3 & {$[6 ; 7 ; 17]$} \\
\hline $\begin{array}{l}\text { Reconhecimento/classificação de } \\
\text { atributos }\end{array}$ & 3 & {$[13 ; 20 ; 23]$} \\
\hline Predição de problemas & 2 & {$[16 ; 22]$} \\
\hline Sistemas baseados no conhecimento & 2 & {$[8 ; 9]$} \\
\hline $\begin{array}{l}\text { Inferir situações/eventos com base } \\
\text { nos dados coletados }\end{array}$ & 1 & {$[15]$} \\
\hline
\end{tabular}

Ao analisarmos o quadro, percebe-se que o processamento e análise dos dados coletados é a maior contribuição de Deep Learning para sistemas IoT (8/24 artigos), seguido pela extração de características (5/24 artigos) e reconhecimento/classificação de padrões visuais por imagens e auxílio em tomadas de decisão (ambos 4/24 artigos). Outras contribuições como reconhecimento/classificação de atributos e melhorias no tráfego da rede corresponderam, cada uma, com 3 dos 24 artigos resultantes do mapeamento.

\subsection{Questão 2: Quais os principais requisitos $e$ princípios aplicados em sistemas que utilizam Deep Learning e IoT conjuntamente?}

Analisando os artigos escolhidos, percebemos que o requisito que mais aparece é a necessidade da existência de vários dados para treinamento dos sistemas que utilizam Deep Learning. Essa necessidade parece ser inerente a qualquer sistema que utiliza de alguma forma Aprendizado de Máquina. Além disso, para o processamento desses dados, se faz necessário máquinas com grande capacidade de processamento.

Outros requisitos e princípios foram encontrados, são eles: dinamicidade, eficiência, produtividade, conectividade, disponibilidade, inteligência, adaptabilidade, ergonomia, integração com outras tecnologias de computação, comunicação, estabilidade, desempenho, confiabilidade, robustez, portabilidade entre plataformas, segurança, manutenibilidade, execução em tempo real e capacidade de generalização. Os sistemas propostos nos artigos utilizam boa parte desses princípios e requisitos em sua fase de estudo e desenvolvimento.

\subsection{Questão 3: Qual(is) a(s) principal(is) arquitetura(s) empregada(s) em sistemas que utilizam Deep Learning e IoT conjuntamente?}

O objetivo dessa questão é descobrir as principais arquiteturas empregadas em sistemas que utilizam Deep Learning e IoT conjuntamente. A partir da pesquisa 
realizada, vimos que a arquitetura mais utilizada é a por camadas (12/24 artigos), onde 6/12 dos artigos são arquiteturas de 3 camadas e os 6/12 restantes são de 4 camadas. Em seguida, temos a arquitetura orientada ao conhecimento (3/24 artigos), que pode ser subdividida em: arquitetura por módulos de raciocínio, cíclica e de gerenciamento (ambas 1/24 artigos). Por fim, temos as arquiteturas baseadas na Internet (1/24 artigos) e em Sistemas Embarcados (1/24). O Quadro 2 apresenta o mapeamento das arquiteturas abordadas pelos artigos.

Quadro 2: Arquiteturas dos sistemas.

\begin{tabular}{|c|c|c|c|}
\hline Arquitetura & Detalhamento & $\#$ & Referências \\
\hline \multirow[b]{2}{*}{ Por camadas } & $\begin{array}{l}3 \text { camadas: } \\
\bullet \quad \text { Física/Aplicação } \\
\bullet \quad \text { Redes } \\
\bullet \quad \text { Inteligência }\end{array}$ & 6 & {$[4 ; 6 ; 13 ; 16 ; 19 ; 23]$} \\
\hline & 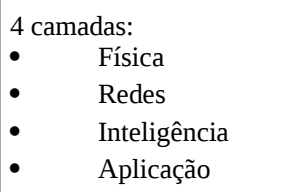 & 6 & {$[1 ; 10 ; 11 ; 21 ; 22 ; 24]$} \\
\hline \multirow{3}{*}{$\begin{array}{l}\text { Orientado ao } \\
\text { conhecimento }\end{array}$} & Módulos de raciocínio & 1 & {$[15]$} \\
\hline & Cíclica & 1 & [9] \\
\hline & Gerenciamento & 1 & {$[8]$} \\
\hline $\begin{array}{l}\text { Baseado na } \\
\text { Internet }\end{array}$ & Redes de computadores & 1 & {$[14]$} \\
\hline $\begin{array}{l}\text { Sistemas } \\
\text { embarcados }\end{array}$ & $\begin{array}{l}\text { Dispositivos de baixa } \\
\text { potência e baixo consumo } \\
\text { de energia }\end{array}$ & 1 & {$[11]$} \\
\hline
\end{tabular}

\subsection{Questão 4: Qual o contexto de uso desses} sistemas?

A pesquisa mostrou que o principal contexto de uso é o reconhecimento de pessoas ou objetos em um ambiente (5/24 artigos), seguido pelo monitoramento de pacientes (4/24 artigos) e pelo monitoramento tanto de estruturas físicas como de uma rede em um sistema IoT (ambos 3/24 artigos). Além desses, existem outros, em menor quantidade, como o monitoramento de motoristas e gestão de pessoas (ambos 2/24 artigos) e o reconhecimento de contexto para os dados dos sensores de IoT e gerenciamento de ambientes (ambos 1/24 artigos). O Quadro 3 mostra o mapeamento dos contextos de uso dos sistemas retornados pela pesquisa.

\subsection{Questão 5: Quais exemplos de aplicações/sistemas existentes?}

Por fim, diferentemente da questão anterior, procuramos por exemplos de sistemas ou aplicações já em uso ou que estão em fase de estudo/desenvolvimento. Sendo assim, nosso estudo mostrou uma variedade de sistemas, entre eles, sistema de cuidados da saúde e para ambientes inteligentes (ambos 4/24 artigos), seguido por sistemas para verificar condições físicas de estruturas e de balanceamento de dados para IoT (ambos 3/24 artigos), além de sistemas de identificação de pessoas em lojas, de monitoramento do comportamento de motoristas e os de informação e conhecimento (ambos 2/24 dos artigos). O Quadro 4 mostra o mapeamento dos sistemas e aplicações já existentes retornados pela pesquisa.
Quadro 3: Contexto de uso dos sistemas.

\begin{tabular}{|l|l|l|}
\hline Contexto & $\#$ & Referências \\
\hline $\begin{array}{l}\text { Reconhecimento de pessoas ou } \\
\text { objetos em um ambiente. }\end{array}$ & 5 & {$[10 ; 11 ; 12 ; 18 ; 19]$} \\
\hline Monitoramento de pacientes & 4 & {$[2 ; 4 ; 21 ; 24]$} \\
\hline Monitoramento de estruturas físicas & 3 & {$[16 ; 20 ; 22]$} \\
\hline $\begin{array}{l}\text { Monitoramento da rede de um } \\
\text { sistema IoT. }\end{array}$ & 3 & {$[6 ; 7 ; 17]$} \\
\hline Monitoramento de motoristas & 2 & {$[1 ; 3]$} \\
\hline Gestão do conhecimento & 2 & {$[8 ; 9]$} \\
\hline $\begin{array}{l}\text { Reconhecimento de contexto para } \\
\text { os dados dos sensores de IoT. }\end{array}$ & 1 & {$[15]$} \\
\hline Gerenciamento de ambientes & 1 & {$[23]$} \\
\hline
\end{tabular}

Quadro 4: Exemplos de aplicações/sistemas.

\begin{tabular}{|l|l|l|}
\hline \multicolumn{1}{|c|}{ Aplicações/Sistemas } & $\#$ & \multicolumn{1}{|c|}{ Referências } \\
\hline Sistema de cuidados da saúde & 4 & {$[2 ; 4 ; 21 ; 24]$} \\
\hline Sistema para ambientes inteligentes & 4 & {$[2 ; 10 ; 19 ; 23]$} \\
\hline $\begin{array}{l}\text { Sistema para verificar condições } \\
\text { físicas de estruturas (geradores } \\
\text { eólicos, estruturas civis e esgotos) }\end{array}$ & 3 & {$[16 ; 20 ; 22]$} \\
\hline $\begin{array}{l}\text { Sistema de balanceamento de dados } \\
\text { para IoT }\end{array}$ & 3 & {$[6 ; 7 ; 17]$} \\
\hline $\begin{array}{l}\text { Sistema de identificação de pessoas } \\
\text { em lojas }\end{array}$ & 2 & {$[5 ; 18]$} \\
\hline $\begin{array}{l}\text { Sistema de monitoramento do } \\
\text { comportamento de motoristas }\end{array}$ & 2 & {$[1 ; 3]$} \\
\hline $\begin{array}{l}\text { Sistema de informação e } \\
\text { conhecimento }\end{array}$ & 2 & {$[8 ; 9]$} \\
\hline Sistema para robôs inteligentes & 1 & {$[2]$} \\
\hline $\begin{array}{l}\text { Sistemas de orientação e } \\
\text { informação de estacionamento }\end{array}$ & 1 & {$[11]$} \\
\hline $\begin{array}{l}\text { Sistema de reconhecimento, em } \\
\text { tempo real, de objetos em uma } \\
\text { imagem }\end{array}$ & 1 & {$[12]$} \\
\hline $\begin{array}{l}\text { Sistema de reconhecimento de } \\
\text { atividades humanas }\end{array}$ & 1 & {$[13]$} \\
\hline $\begin{array}{l}\text { Sistema de raciocínio e inferência } \\
\text { de situações }\end{array}$ & {$[15]$} \\
\hline
\end{tabular}

\section{Conclusão}

Avaliar todos os métodos e abordagens da Inteligência Artificial (IA) que podem ser trabalhados em conjunto com IoT, e vice-versa, é uma atividade bastante complexa, pois tanto a área de IA quanto a de IoT trabalham com diversas possibilidades e ferramentas. Nesse contexto, este trabalho realizou um mapeamento sistemático de literatura no intuito de conhecer como funcionam os sistemas que utilizam Deep Learning e IoT conjuntamente, limitando assim o escopo de estudo.

Conclui-se também que diversos sistemas e aplicações que utilizam IoT e Deep Learning conjuntamente já estão em fase de desenvolvimento ou de estudo e que a união dessas duas tecnologias é bem promissora para diversas atividades do cotidiano. 


\section{Bibliografia}

[1] Virmani, S. \& Gite, S. (2017). Performance of Convolutional Neural Network and Recurrent Neural Network for anticipation of driver's conduct. In Anais do IEEE. DOI: 10.1109/ICCCNT.2017.8204039.

[2] Chen, M., Herrera, F. \& Hwang, K. (2017). Cognitive Computing: Human-Centered Computing with Cognitive Intelligence on Clouds. In Anais do IEEE. DOI: 10.1109/ACCESS.2018.2791469.

[3] Streiffer, C., Raghavendra, R., Benson, T. \& Mudhakar Srivatsa, M. (2017). DarNet: A Deep Learning Solution for Distracted Driving Detection. In Anais da ACM. DOI: $10.1145 / 3154448.3154452$.

[4] Morshed, A., Prakash Jayaraman, P., Sellis, T., Georgakopoulos, D., Villari, M. \& Ranjan, R. (2017). Deep Osmosis: Holistic Distributed Deep Learning in Osmotic Computing in Anais IEEE. DOI: 10.1109/MCC.2018.1081070.

[5] Li, P., Chen, Z., T. Yang, L., Zhang, Q. \& Jamal Deen, M. (2017). Deep Convolutional Computation Model for Feature Learning on Big Data in Internet of Things. In Anais do IEEE. DOI: 10.1109/TII.2017.2739340.

[6] Li, H., Ota, K. \& Dong, M. (2018). Learning IoT in Edge: Deep Learning for the Internet of Things with Edge Computing. In Anais do IEEE. DOI: 10.1109/MNET.2018.1700202.

[7] Zhu, J., Song, Y., Jiang, D. \& Song, H. (2017). A New Deep-Q-Learning-Based Transmission Scheduling Mechanism for the Cognitive Internet of Things. In Anais do IEEE. DOI: 10.1109/JIOT.2017.2759728.

[8] Zhang, H., Li, F., Wang, J., Wang, Z., Sanín, C. \& Szczerbicki, E. (2017). Experience-Oriented Intelligence for Internet of Things. In Anais do Cybernetics and Systems. DOI: 10.1080/01969722.2016.1276771.

[9] Yu, J., Kim, Y.-M., Kwon, S., Kim, K., Kim, N.-S., Kim, S.-J. \& Pyo, C.-S. (2017). A Study on the Virtuous Circle Self-Learning Methods for Knowledge Enhancement. In Anais da 2017 International Conference on Platform Technology and Service, PlatCon $2017 \quad$ - Proceedings. DOI: 10.1109/PlatCon.2017.7883683.

[10] Li, J., Wang, X. \& Su, H. (2016). Supermarket commodity identification using convolutional neural networks. In Anais da Proceedings of 2016 2nd International Conference on Cloud Computing and Internet of Things, CCIOT 2016. DOI: 10.1109/CCIOT.2016.7868315.

[11] Valipour, S., Siam, M., Stroulia, E. \& Jagersand, M. (2016). Parking-stall vacancy indicator system, based on deep convolutional neural networks. In Anais do 2016 IEEE 3rd World Forum on Internet of Things, WF-IoT 2016. DOI: 10.1109/WF-IoT.2016.7845408.

[12] Valipour, S., Siam, M., Stroulia, E. \& Jagersand, M. (2017). Enabling Deep Learning on IoT Devices. In Anais do IEEE. DOI: 10.1109/MC.2017.3641648.

[13] Ravi, D., Wong, C., Lo, B. \& Yang, G.-Z. (2017). A Deep Learning Approach to on-Node Sensor Data Analytics for Mobile or Wearable Devices. In Anais do IEEE. DOI: 10.1109/JBHI.2016.2633287.

[14] Liu, F., Shi, Y. \& Li, P. (2017). Analysis of the Relation between Artificial Intelligence and the Internet from the Perspective of Brain Science. In Anais da Procedia Computer Science. DOI: 10.1016/j.procs.2017.11.383.

[15] Park, S., Sohn, M., Jin, H. \& Lee, H. (2016). Situation reasoning framework for the Internet of Things environments using deep learning results. In Anais do IEEE. DOI: 10.1109/ICKEA.2016.7803006.

[16] Chen, F., Fu, Z. \& Yang. Z. (2018). Wind power generation fault diagnosis based on deep learning model in internet of things (IoT) with clusters. In Anais do Springer Science+Business Media, LLC, part of Springer Nature.DOI: https://doi.org/10.1007/s10586-018-2171-6.
[17] Kim, H. \& Kim, J.(2016). A load balancing scheme based on deep-learning in IoT. In Anais Springer Science+Business Media New York. DOI: https://doi.org/10.1007/s10586-016-0667-5.

[18] Ho Oh, S., Kim, G. \& Lim, K. (2017). Compact deep learned feature-based face recognition for Visual Internet of Things. In Anais Springer Science+Business Media, LLC, part of Springer Nature. DOI: https://doi.org/10.1007/s11227-017-2198-0.

[19] Liu, W., Clarence Yan, C., Liu, J. \& Ma1, H.(2017). Deep learning based basketball video analysis for intelligent arena application. In Anais Springer Science+Business Media, LLC. DOI: https://doi.org/10.1007/s11042-017-5002-5.

[20] Guo, J., Xie, X., Bie, R. \& Sun, L. (2014). Structural health monitoring by using a sparse coding-based deep learning algorithm with wireless sensor networks. In Anais Personal and Ubiquitous Computing. DOI: https://doi.org/10.1007/s00779-014-0800-5.

[21] Shi, X., Hao, Y., Zeng, D., Wang, L., Hossain, S., Md Mizanur Rahman, S. \& Alelaiwi, A. (2016). CloudAssisted Mood Fatigue Detection System. In Anais do Springer. DOI: https://doi.org/10.1007/s11036-0160757-x.

[22] Zhang, D., Martinez, N., Lindholm, G. \& Ratnaweera, H. (2018). Manage Sewer In-Line Storage Control Using Hydraulic Model and Recurrent Neural Network. In Anais Springer Science+Business Media B.V., part of Springer Nature. DOI: https://doi.org/10.1007/s11269018-1919-3.

[23] Chen, G., Wang, A., Zhao, S., Liu, L. \& Chang, C. (2017). Latent feature learning for activity recognition using simple sensors in smart homes. In Anais do Springer Science+Business Media, LLC. DOI: https://doi.org/10.1007/s11042-017-5100-4.

[24] Singh, H., Yadav, G., Mallaiah, R., Joshi, P., Joshi, V., Kaur, R., Bansal, S. \& K. Brahmachari, S. (2017). iNICU - Integrated Neonatal Care Unit: Capturing Neonatal Journey in an Intelligent Data Way, In Anais Journal of Medical Systems. DOI: https://doi.org/10.1007/s10916-017-0774-8.

[25] Wortmann, F. \& Flüchter, K. (2015). Internet of Things: Technology and Value Added.

[26] LeCun, Y., Bengio, Y. \& Hinton, G. (2015). Deep Learning: Review. 